\title{
HPV infection, cervical abnormalities, and cancer in HIV-infected women in Mumbai, India: I 2-month follow-up
}

This article was published in the following Dove Press journal:

International Journal of Women's Health

12 August 2013

Number of times this article has been viewed

\section{Petros Isaakidis ${ }^{1,2}$ \\ Sharmila Pimple ${ }^{3}$ \\ Bhanumati Varghese' \\ Samsuddin Khan' \\ Homa Mansoor' \\ Joanna Ladomirska' \\ Neelakumari Sharma' \\ Esdras Da Silva' \\ Carol Metcalf ${ }^{4}$ \\ Severine Caluwaerts ${ }^{4}$ \\ Petra Alders ${ }^{4}$ \\ Evangelia E Ntzani² \\ Tony Reid ${ }^{4}$}

'Médecins Sans Frontières, Mumbai, India; ${ }^{2} \mathrm{Clinical}$ and Molecular Epidemiology Unit, Department of Hygiene and Epidemiology, University of Ioannina School of Medicine, loannina, Greece; ${ }^{3}$ Preventive Oncology Department, Tata Memorial Hospital, Mumbai, India; ${ }^{4}$ Médecins Sans Frontières, Brussels, Belgium
Correspondence: Petros Isaakidis Médecins Sans Frontières, Chandni Bungalow, Union Park, Off Carter Road, Khar (W), Mumbai 400 052, India $\mathrm{Tel}+9$ | 99305342 I I

Email msfocb-asia-epidemio@brussels. msf.org
Background: HIV-infected women are at a higher risk of cervical intraepithelial neoplasia (CIN) and cancer than women in the general population, partly due to a high prevalence of persistent human papillomavirus (HPV) infection. The aim of the study was to assess the burden of HPV infection, cervical abnormalities, and cervical cancer among a cohort of HIV-infected women as part of a routine screening in an urban overpopulated slum setting in Mumbai, India.

Methods: From May 2010 to October 2010, Médecins Sans Frontières and Tata Memorial Hospital Mumbai offered routine annual Pap smears and HPV DNA testing of women attending an antiretroviral therapy (ART) clinic and a 12-month follow-up. Women with abnormal test results were offered cervical biopsy and treatment, including treatment for sexually transmitted infections (STIs).

Results: Ninety-five women were screened. Median age was 38 years (IQR: 33-41); median nadir CD4-count 143 cells/ $\mu \mathrm{L}$ (IQR: 79-270); and median time on ART 23 months (IQR:10-41). HPV DNA was detected in 30/94 women (32\%), and 18/94 (19\%) showed either low-grade or high-grade squamous intraepithelial lesions (LSIL/HSIL) on Pap smear. Overall, $>50 \%$ had cervical inflammatory reactions including STIs. Of the 43 women with a cervical biopsy, eight (8.4\%) had CIN-1, five (5.3\%) CIN-2, and two (2.1\%) carcinoma in situ. All but one had HPV DNA detected (risk ratio: 11, 95\% confidence interval: 3.3-34). By October 2011, 56 women had completed the 12-month follow-up and had been rescreened. No new cases of HPV infection/LSIL/HSIL were detected.

Conclusion: The high prevalence of HPV infection, STIs, and cervical lesions among women attending an ART clinic demonstrates a need for routine screening. Simple, one-stop screening strategies are needed. The optimal screening interval, especially when resources are limited, needs to be determined.

Keywords: HIV/AIDS, HPV, women's health, cervical cancer, operational research, India

\section{Introduction}

About half a million cases of cervical cancer occur annually worldwide, $80 \%$ of which occur in low-resource countries. ${ }^{1}$ In India, cervical cancer ranks as the most frequent cancer among women, especially among those between 15 and 45 years of age. ${ }^{2}$ Human papillomavirus (HPV) is a major risk factor for cervical cancer since as much as $83 \%$ of invasive cervical cancers are associated with human papillomavirus 16 or 18, and about $8 \%$ of women in the general population are estimated to harbor cervical HPV infection at a given time. ${ }^{2}$

HIV-infected women in various settings were found to have higher rates of persistent HPV infection, more abnormal Pap tests, more cervical intraepithelial neoplasia 
(CIN) lesions, and aggressive cervical cancer, compared with the general population, and these numbers are increasing. ${ }^{3-6}$ The high rate of cervical disease reported in HIV-infected women and the extended life expectancy due to better access to antiretroviral therapy (ART) have led to recommendations for aggressive screening and prompt treatment of cervical lesions; yet, the most appropriate strategy to screen HIVinfected women, particularly in resource-constrained settings, remains unclear. ${ }^{7-9}$ Moreover, the existing evidence on the impact of ART on the incidence and evolution of CIN is still unclear, thus reinforcing the recommendation of systematic screening of all HIV-infected women, even those on ART., ${ }^{3,6}$ Overall, the evidence assessing the extent and magnitude of HPV infection and the burden of cervical abnormalities and cancer among HIV-infected women is sparse and remains largely undocumented.

The aim of this study was to assess the burden of HPV infection, cervical abnormalities, and cervical cancer among a cohort of HIV-infected women as part of a routine screening in an urban, overpopulated slum setting in Mumbai, India. Specific objectives included: to measure the overall yield of HPV infection, cervical abnormalities, and cervical cancer; to report on the treatment outcomes among women with treated abnormalities; and to identify factors associated with cervical abnormalities, especially factors related to the HIV infection (CD4 count, ART, etc). To our knowledge, this is one of the first reports on cervical abnormalities in HIV-infected patients in India.

\section{Methods}

\section{Study design}

This was a prospective cohort of HIV-infected patients followed up from February 2010 to October 2011.

\section{Setting and study population}

Médecins Sans Frontières (MSF) has been operating a specialized HIV clinic in Mumbai, India since 2006, providing treatment free of charge to patients referred by ART centers from the greater Mumbai area and by community non-governmental organizations. Patients are referred to the clinic because of coinfections (especially multidrugresistant tuberculosis and hepatitis B or C) and because they require antiretroviral agents or regimens not readily available through the national ART program. Most of the patients are slum dwellers.

A universal cervical cancer screening was organized, in collaboration with the Preventive Oncology Department of the Tata Memorial Hospital, Mumbai, India, from February to October 2010. HIV-infected women aged 25-65 years with intact uterus and with no past history of cervical neoplasia and who gave consent were recruited. Women coinfected with multidrug-resistant tuberculosis at the early stages of treatment were offered the screening at a later stage, depending on their condition. Women that refused consent were further excluded from the study.

\section{Pap test, HPV detection, and cervical biopsy}

All the women participating in the study were tested for cervical cytology and HPV and underwent colposcopy with or without biopsy. HPV testing by Hybrid capture II was done by cervical sampling brush (Digene cervical sampler, Qiagen, Venlo, the Netherlands). HPV DNA status for high-risk HPV types $(16,18,31,33,35,39,45,51,52,56,58,59$, and 68) was determined with the use of the second-generation HC II probe B, microtiter assay (Digene). Colposcopy was carried out by trained doctors for all participants irrespective of their screening test result. Colposcopy-guided punch biopsies were obtained from subjects with abnormal findings on colposcopy. Biopsy specimens were processed and reported using the CIN system. True disease status was defined as CIN-2 and worse lesions. CD4 T-cell counts (BD, Franklin Lakes, NJ, USA) were obtained from the participant's medical record. Nadir CD4 T-cell count was defined as the lowest level of immunosuppression recorded for a specific patient.

\section{Treatment protocol and follow-up}

Women with any grade of CIN were called back for cryotherapy or loop electrosurgical excision procedure (LEEP), as appropriate. Women with suspected invasive cancer were referred to the Tata Memorial Hospital for further management. All treated women were followed-up for up to 1 year for colposcopy evaluation in order to determine cure rates.

\section{Data collection and statistical methods}

Information on HIV and ART of all patients was prospectively collected in specifically designed patient files and routinely entered in a database. For the HPV and cervical cancer screening, clinical and laboratory information were recorded in the same patient files but entered in a different database. A unique identification code was assigned to each patient and was used in both databases. Patient characteristics were described using medians, interquartile ranges (IQRs), and percentages, as appropriate. We estimated the burden of cervical abnormalities at the screening and follow-up using proportions. 
In order to identify predictors for cervical pathologies, univariate and multivariate analyses were performed using logistic regression and generalized linear models. Multivariate models considered all variables with $P<0.1$ on univariate models and used a backward elimination approach for final selection. There was no overt violation of the proportionality assumption. Statistical analyses were performed using SPSS (v 16.0; IBM Corporation, Armonk, NY, USA) and Stata (version 11; StataCorp LP, College Station, TX, USA).

\section{Ethics}

The tests, follow-up of abnormalities, and treatment options were explained to patients, and their written consent requested. The study met the MSF's Ethics Review Board-approved criteria for analysis of routinely collected program data.

\section{Results}

\section{Baseline patient characteristics and HIV status}

Of the 390 HIV-infected women registered in the HIV clinic from 2006, the 99 adult women who attended the clinic from October to May 2010 were considered for inclusion in the study. Three women denied screening and one teenager reported no history of sexual activity and was excluded from the study; thus, 95 women were screened during the first screening. The median age was 38 (IQR: 33-41), and 51\% of the women were aged between 30 and 39 years (Table 1). Approximately half of the women were married or living with a partner at the time of the study; a large proportion of widows and separated women was recorded in this cohort (46\%). Most women had attained at least primary education, while 13\% had received higher education. Eighty percent had either never smoked or were not current smokers, while $98 \%$ of the cohort had never used drugs or alcohol. A large majority (98.9\%) of the women were not making use of any type of hormonal contraceptive method. Forty-five percent of the women had had two or more pregnancies during their lifetime. Thirty-seven percent had a nadir CD4 T-cell count of $<100$ cells/ $\mu \mathrm{L}$. All patients were on ART at the time of the screening; the median time on ART was 23 months (IQR: 10-41).

\section{Baseline HPV infection status, cytology and pathology results, and treatment}

The majority (98\%) of this cohort of HIV-infected women had never had a gynecological examination before the screening was offered. For one woman, a Pap smear test result was not
Table I Sociodemographic characteristics of the Mumbai HIVinfected female cohort

\begin{tabular}{|c|c|c|}
\hline Characteristic (N) & n (\%) & Median (IQR) \\
\hline Age (years) (95) & & $38(33-4 I)$ \\
\hline$<30$ & $12(12.6)$ & \\
\hline $30-39$ & $48(50.5)$ & \\
\hline$\geq 40$ & $35(36.8)$ & \\
\hline Nadir CD4 count (cells/ $\mu \mathrm{L}$ ) (95) & & | 43 (79-270) \\
\hline$\leq 100$ & $35(36.8)$ & \\
\hline $10 \mid-200$ & $26(27.4)$ & \\
\hline $20 I-350$ & $16(16.8)$ & \\
\hline$>350$ & $18(19.0)$ & \\
\hline Time on ART (months) (95) & & $23(|0-4|)$ \\
\hline $0-11$ & $25(26.3)$ & \\
\hline $12-23$ & $23(24.2)$ & \\
\hline $24-35$ & $18(19.0)$ & \\
\hline$\geq 36$ & $29(30.5)$ & \\
\hline \multicolumn{3}{|l|}{ Education (95) } \\
\hline Illiterate & $24(25.3)$ & \\
\hline Primary & $19(20.0)$ & \\
\hline Secondary & $40(42.1)$ & \\
\hline Tertiary & $12(12.6)$ & \\
\hline Monthly income (USD) (95) & & $60(40-100)$ \\
\hline$<50$ & $34(35.8)$ & \\
\hline $50-99$ & $33(34.7)$ & \\
\hline$\geq 100$ & $28(29.5)$ & \\
\hline Gravidity (94) & & $\mathrm{I}(\mathrm{I}-2)$ \\
\hline 0 & $10(10.6)$ & \\
\hline I & $42(44.7)$ & \\
\hline$\geq 2$ & $42(44.7)$ & \\
\hline \multicolumn{3}{|c|}{ Married/currently living with partner (95) } \\
\hline Yes & $48(50.6)$ & \\
\hline No & $47(49.4)$ & \\
\hline \multicolumn{3}{|l|}{ Ever smoked/chewed tobacco (95) } \\
\hline Yes & $19(20)$ & \\
\hline No & $76(80)$ & \\
\hline \multicolumn{3}{|l|}{ Ever used drugs/alcohol (95) } \\
\hline Yes & $2(2.1)$ & \\
\hline No & $93(97.9)$ & \\
\hline \multicolumn{3}{|l|}{ Use of hormonal contraceptives (94) } \\
\hline Yes & $\mathrm{I}(\mathrm{I} . \mathrm{I})$ & \\
\hline No & $93(98.9)$ & \\
\hline
\end{tabular}

Abbreviations: ART, antiretroviral therapy; IQR, interquartile range; USD, US dollar.

retrieved. HPV infection was detected in $30(32 \%)$ of the 94 women with an available result.

Overall, 43 women, almost half of the participants, had cervical inflammatory reactions. Forty-one percent had inflammation of the cervix, including STIs, but not intraepithelial lesions. Cervical cytology was abnormal in 18 of 94 women (19\%). Low-grade squamous intraepithelial lesions (LSIL) and high-grade squamous intraepithelial lesions (HSIL) were detected in $14 \%$ and $5 \%$ of the participants, respectively. 
The women with cervical inflammatory reactions were referred for colposcopy with a guided cervical biopsy by the examining physician during the first round of screening. Biopsy was abnormal in 16\% women: eight ( $8 \%$ ) had CIN-1, five (5\%) CIN-2, and two (2\%) women were diagnosed with carcinoma in situ.

Five patients underwent LEEP during the first screening round; four women with CIN-2 and one with CIN-1. Eight women received cryotherapy: one woman with $\mathrm{CIN}-2$ and seven women with CIN-1. One woman with cervical cancer died in a car accident before any treatment was started. The second woman diagnosed with cervical cancer was successfully operated on and received radiotherapy and chemotherapy as per the recommended treatment guidelines. This patient, at the 18-month follow-up, had to switch to second-line ART due to virological failure after 3 years on first-line ART. The patient was, however, in excellent general condition and she was not immunologically compromised at the time of the ART switch.

\section{2-month follow-up}

Figure 1 shows the flow of patients in the two screening rounds and reasons for not screening. Sixteen women were not eligible for rescreening as they hadn't completed 12 months of follow-up at the time of the analysis. By October 2011, 56 (59\%) women had completed at least

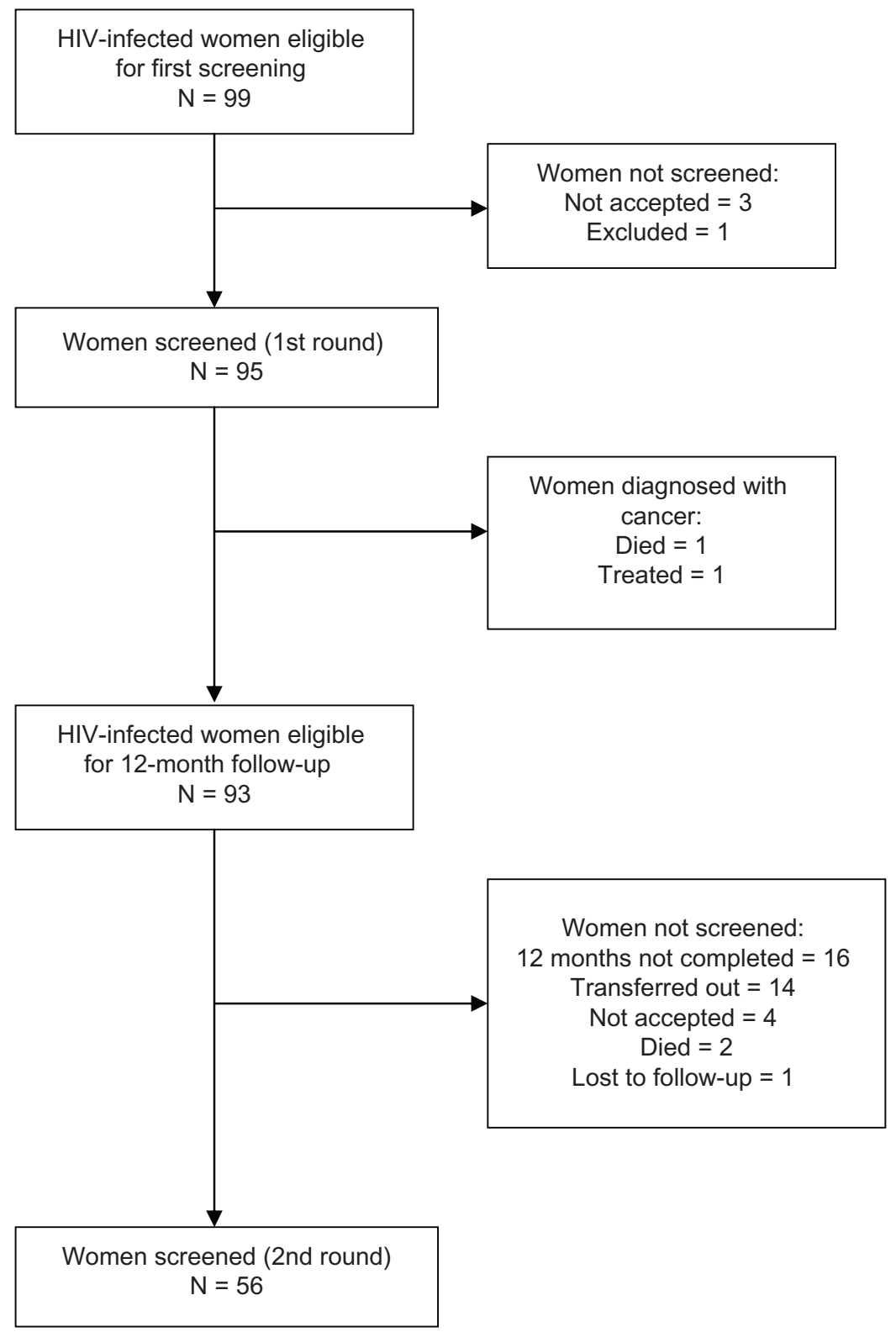

Figure I Flowchart of cervical cancer screening in a cohort of HIV-infected women at baseline and I2-month follow-up. 
12 months of follow-up and had been rescreened. No new cases of HPV infection, LSIL, or HSIL were detected on rescreening. Of the 15 women who were found HPV-positive in the first screening and pursued a second screening, nine tested negative in the second screening while the remaining six remained HPV-positive in the second screening at 12 months.

\section{Factors associated with HPV infection and CIN}

In the bivariate regression analysis, no factor was nominally significantly associated with an increased risk for HPV infection. In multivariate models, and adjusting for all factors, nadir CD4, time on treatment, and parity were associated with HPV infection (Table 2).

All but one of the eleven women (91\%) with CIN or cancer were infected with HPV. Similarly, the two participants with invasive cervical cancer were infected with HPV. In the bivariate models, and despite the lack of power, HPV infection was significantly associated with cervical neoplasia (RR: 11, 95\% CI: 3.3-34). In further

Table 2 Factors associated with HPV infection and cervical abnormalities $(\mathrm{N}=93)$

\begin{tabular}{|c|c|c|c|}
\hline Factor & n (\%) & $\begin{array}{l}\text { HPV } \\
\text { OR } \\
\text { (P-value) }\end{array}$ & $\begin{array}{l}\text { High- and low- } \\
\text { grade squamous } \\
\text { intraepithelial } \\
\text { lesions } \\
\text { OR ( } P \text {-value) }\end{array}$ \\
\hline \multicolumn{4}{|l|}{ Age (years) } \\
\hline$<35$ & $29(30.5)$ & & \\
\hline$\geq 35$ & $66(69.5)$ & $0.46(0.203)$ & $0.68(0.613)$ \\
\hline \multicolumn{4}{|l|}{ Nadir CD4 (cells/ $\mu \mathrm{L})$} \\
\hline$\leq 200$ & $6 I(64.2)$ & $3.73(0.025)^{*}$ & $1.04(0.954)$ \\
\hline$>200$ & $34(35.8)$ & & \\
\hline \multicolumn{4}{|l|}{ Months on ART } \\
\hline$<12$ & $25(26.3)$ & $3.57(0.027)^{*}$ & $1.27(0.743)$ \\
\hline$\geq 12$ & $70(73.7)$ & & \\
\hline \multicolumn{4}{|l|}{ Previous pregnancies ${ }^{\dagger}$} \\
\hline 0 or 1 & $52(54.7)$ & & \\
\hline$\geq 2$ & $42(44.2)$ & $3.32(0.035)^{*}$ & $0.88(0.859)$ \\
\hline \multicolumn{4}{|l|}{ Income (USD/month) } \\
\hline$<50$ & $34(35.8)$ & $\mathrm{I} .33(0.580)$ & $0.90(0.882)$ \\
\hline$\geq 50$ & $61(64.2)$ & & \\
\hline \multicolumn{4}{|l|}{ Level of education } \\
\hline Primary or less & $43(45.3)$ & $1.29(0.611)$ & $1.05(0.934)$ \\
\hline Secondary or more & $52(54.7)$ & & \\
\hline \multicolumn{4}{|l|}{ HPV at baseline ${ }^{\dagger}$} \\
\hline Not detected & $64(67.4)$ & - & \\
\hline Detected & $30(31.6)$ & - & $19.69(<0.001)^{*}$ \\
\hline
\end{tabular}

Notes: $* P<0.05$; 994 patients were included.

Abbreviations: ART, antiretroviral therapy; HPV, human papillomavirus; OR, odds ratio. multivariate models, no sociodemographic or clinical factor was found to be significantly associated with cervical neoplasia (Table 2).

\section{Discussion}

In the present study, we estimated the prevalence and 12-month incidence of cervical abnormalities and assessed factors associated with cervical neoplasia among HIVinfected women enrolled in care in an HIV clinic in Mumbai, India who attended a screening program. The vast majority of women had never previously been screened for cervical abnormalities. We found that one-third of the women tested positive for HPV; more than half had inflammation of the cervix, including STIs; and as many as $17 \%$ of the women screened had abnormal Pap smear results. Prevalence of CIN was similarly high, at approximately $16 \%$. Finally, we found no new cases of HPV infection, LSIL, HSIL, CIN, or cancer among women rescreened 12 months after their initial screening.

Studies among the general population in resource-limited countries have shown high prevalence of cervical neoplasia. However, little is known about the prevalence and incidence of cervical abnormalities and HPV infection among HIVinfected women. Interestingly, our study findings are in accordance with a very recent study in which the investigators observed similar rates of HPV infection and cervical pathology. ${ }^{10}$ Moreover, in studies among HIV-infected women in Sao Paolo, Rio De Janeiro, and Belo Horizonte, Brazil, $13 \%, 24 \%$, and $24 \%$ had a diagnosis of squamous intraepithelial lesions, respectively. ${ }^{11-13}$ In studies in Cambodia, Thailand, and People's Republic of China, the prevalence of HSIL/LSIL among women attending HIV clinics was $17 \%, 16 \%$, and $8 \%$, respectively. ${ }^{8,14,15}$ Studies conducted in Zambia and Kenya reported higher prevalences, 33\% and $27 \%$, respectively, among HIV-infected women ${ }^{16,17}$ Finally, in our study, and bearing in mind the available published evidence, we report an intermediate HPV prevalence among HIV-infected women.

It is well established that HPV infection is a contributor to cervical cancer and that it progresses to squamous intraepithelial neoplasia more frequently and rapidly in HIV-infected women than in the general population. ${ }^{18}$ Our main study findings indicate that HPV infection was frequent in this cohort of patients and significantly associated with squamous intraepithelial lesions, suggesting that this population follows an epidemiological pattern in common with other HPV-infected women, who remain at high risk for cervical neoplasia; as such, aggressive screening and management are justified 
for HIV/ HPV co-infected women. We offered colposcopy plus biopsy to all women with clinical findings suggestive of neoplasia as well as to all women with abnormal Pap smears. This practice proved very effective for the confirmation of cervical abnormalities, and guided prompt treatment. Nevertheless, the estimated uptake of Pap tests in India is discouragingly low, at $2.6 \%$; the situation is no better in urban centers, where uptake is only slightly higher, at $4.9 \%{ }^{2}$ Our experience from Mumbai, a major metropolitan center with access to HPV DNA testing and quality-controlled cytology and pathology showed that the very availability of such services is not enough to guarantee increased access. Major constraints include the capacity of the existing facilities and lack of awareness in both the general population and among health care providers. Our findings suggest that, in settings with limited access to care, women with a history of HPV should be prioritized, especially if HIV-infected.

Modeling for factors potentially associated with squamous intraepithelial lesions in HIV-infected women, we found that immunosuppression, as measured by nadir CD4 count, was not significantly associated with squamous intraepithelial lesion prevalence. Similarly, time on ART was not found to have a statistically significant protective effect in the occurrence of squamous intraepithelial lesions in this population. The absence of significant associations could be explained by the lack of power to detect these associations. Nevertheless, the degree of immunosuppression is expected to have an effect on the incidence and progression of squamous intraepithelial lesions among HIV-infected women as it has been shown in several observational studies. ${ }^{13-17}$ To date, evidence on an independent association between ART and the natural history of HPV infection or the risk of squamous intraepithelial lesions and cervical cancer is contradictory and inconclusive. ${ }^{18-21}$ It is likely that the increased survival of these women due to ART may lead to higher incidence and mortality from cervical pathologies and cancer.

There are a number of limitations to this study. First, we fully acknowledge that this is a small study; however, we argue that the setting in which the study took place poses challenges in gathering large sample sizes, and even studying small datasets provides valuable information. Second, we acknowledge that only $60 \%$ of the women were followed up after 12 months. The actual loss to follow-up, death rate, and refusals were relatively low. A significant number of women were transferred out from the program and a relatively large number of women were yet to complete 12 months of follow-up at the time of the analysis. Moreover, we were not able to collect data on the age of first sexual intercourse and the number of lifetime sexual partners. However, India is a conservative society in which extramarital sex is taboo, especially for women, and we would expect a rather homogenous sample in these regards.

Worth discussing is that we found no new cases of HPV infection or squamous intraepithelial lesion in the 12-month follow-up in this cohort of patients. While we strongly believe that screening and management of cervical abnormalities and cancer in HIV-infected women should be recurrent and aggressive, we also acknowledge that, when resources are limited, the type and frequency of screening needs to be carefully determined. United States cervical cancer screening guidelines for HIV-uninfected women 30 years or older were recently revised, with the suggested interval between Pap tests increasing from 3 to 5 years for those with normal cervical cytology results and negative oncogenic HPV. ${ }^{22,23}$ It is not clear if an interval of 3 or 5 or more years between screenings could be used in HIV-infected women who are HPV-negative and cytologically normal. Meanwhile, as HPV testing is not available in most resource-constrained settings, we advocate for annual screening and prompt management of cervical abnormalities. Implementation of a cytology-based screening strategy in rural or resource-limited settings may be challenging. In previous MSF experience in Cambodia, "screening for cervical cancer using the conventional Pap test proved just as difficult for HIV-positive women, already enrolled in care, as for women of the general population." 8

The single-visit screen-and-treat approach, using visual inspection with acetic acid (VIA) and cryotherapy, has been shown to be effective and acceptable among women in resource-constrained settings. However, it is still not well known whether this approach, is effective and safe for HIVinfected women. ${ }^{24,25}$ Studies from Zambia have shown that linking cervical cancer prevention and treatment services (including LEEP) with HIV care and treatment is feasible and safe. ${ }^{26,27}$ Sankaranarayanan et al, in 2009, demonstrated in a cluster-randomized study in rural India that a "single round of HPV testing was associated with a significant reduction in the numbers of advanced cervical cancers and deaths from cervical cancer." ${ }^{28}$ The HIV status of the women enrolled in that study, however, was not discussed in the study report.

Recently, in a large observational study in, USA, it was found that there were no differences in the 5-year cumulative incidence of HSIL and CIN-2 between HIV-infected and -uninfected women. ${ }^{29}$ The authors, however, concluded that 
further evidence is needed, including randomized evidence, before expanding the current recommendations for testing all HIV-infected women for HPV. In resource-limited settings, operational research is also needed to demonstrate the integration of cervical cancer prevention within HIV programs.

\section{Conclusion}

The high prevalence of HPV infection, STIs, and cervical lesions detected among women attending an ART clinic in Mumbai, India demonstrates a need for routine cervical screening of all HIV-infected women in this setting. We suggest further investigation of simple, preferably one-stop, cervical cancer screening strategies and models for HIVinfected women. Large cohort studies are also needed to determine the optimal screening interval, especially when resources are limited.

\section{Acknowledgments}

The authors wish to acknowledge the contribution of health care workers from the MSF clinic and Tata Memorial Hospital, as well as the patients and their families.

\section{Author contributions}

PI conceived the study and wrote the first study protocol. PI, SP, JL, BV, and SK designed the study. SP, BV, SK, HM, NS, and EDS collected the data. PI, CM, and EEN analyzed the data. PI and EEN drafted the manuscript, and edited multiple versions with inputs from SP, BV, SK, SC, PA, JL, and TR. All authors reviewed and approved the final version of the manuscript.

\section{Disclosure}

The authors report no conflicts of interest in this work.

\section{References}

1. Parkin DM, Stjernswärd J, Muir CS. Estimates of the worldwide frequency of twelve major cancers. Bull World Health Organ. 1984;62(2):163-182.

2. Human Papillomavirus and Related Cancers. Summary Report Update. India. WHO/ICO HPV Information Center; 2010. Available from: http:// www.hpvcentre.net/statistics/reports/IND.pdf. Accessed July 10, 2013.

3. Palefsky JM. Cervical human papillomavirus infection and cervical intraepithelial neoplasia in women positive for human immunodeficiency virus in the era of highly active antiretroviral therapy. Curr Opin Oncol. 2003;15(5):382-388.

4. Serraino D, Carrieri P, Pradier C, et al. Risk of invasive cervical cancer among women with, or at risk for, HIV infection. Int J Cancer. 1999; 30(82):334-337.

5. Pantanowitz L, Michelow P. Review of human immunodeficiency virus (HIV) and squamous lesions of the uterine cervix. Diagn Cytopathol. 2011;39(1):65-72.
6. De Vuyst H, Franceschi S. Human papillomavirus vaccines in HIV-positive men and women. Curr Opin Oncol. 2007;19(5): 470-475.

7. Comprehensive Cervical Cancer Control: A Guide to Essential Practice. Geneva: World Health Organization; 2006.

8. Raguenaud ME, Isaakidis P, Ping C, Reid T. Screening for and treating cervical cancer in HIV-positive women in Cambodia. J Acquir Immune Defic Syndr. 2013;51(5):644-646.

9. Danso D, Lyons F, Bradbeer C. Cervical screening and management of cervical intraepithelial neoplasia in HIV-positive women. Int J STD AIDS. 2006;17:579-586.

10. Joshi S, Sankaranarayanan R, Muwonge R, Kulkarni V, Somanathan T, Divate U. Screening of cervical neoplasia in HIV-infected women in India. AIDS. 2013;27:607-615.

11. Calore EE, Pereira SM, Cavaliere MJ. Progression of cervical lesions in HIV seropositive women: a cytological study. Diagn Cytopathol. 2001;24:117-119.

12. Luz PM, Velasque L, Friedman RK, et al. Cervical cytological abnormalities and factors associated with high-grade squamous intraepithelial lesions among HIV-infected women from Rio de Janeiro, Brazil. Int J STD AIDS. 2012;23:12-17.

13. Teixeira NC, Araújo AC, Correa CM, et al. Prevalence and risk factors for cervical intraepithelial neoplasia among HIV-infected women. Braz J Infect Dis. 2012;16(2):164-169.

14. Mangclaviraj S, Kerr SJ, Chaithongwongwatthana S, et al. Nadir CD4 count and monthly income predict cervical squamous cell abnormalities in HIV-positive women in a resource-limited setting. Int J STD AIDS. 2008;19(8):529-532.

15. Zhang HY, Tiggelaar SM, Sahasrabuddhe VV, et al. HPV prevalence and cervical intraepithelial neoplasia among HIV-infected women in Yunnan Province, People's Republic of China: a pilot study. Asian Pac J Cancer Prev. 2012;13(1):91-96.

16. Lehtovirta P, Paavonen J, Heikinheimo O. Risk factors, diagnosis and prognosis of cervical intraepithelial neoplasia among HIV-infected women. Int J STD AIDS. 2008;19:37-41.

17. Memiah P, Mbuthia W, Kiiru G, et al. Prevalence and risk factors associated with precancerous cervical cancer lesions among HIV-infected women in resource-limited settings. AIDS Res Treat. 2012;2012:953743.

18. Heard I, Tassie JM, Kazatchkine MD, Orth G. Highly active antiretroviral therapy enhances regression of cervical intraepithelial neoplasia in HIV-seropositive women. AIDS. 2002;16: 1799-1802.

19. Mbulawa ZZ, Marais DJ, Johnson LF, Coetzee D, Williamson AL. Impact of human immunodeficiency virus on the natural history of human papillomavirus genital infection in South African men and women. J Infect Dis. 2012;206(1):15-27.

20. Xi LF, Kiviat NB. Cervical neoplasia and highly active antiretroviral therapy. J Natl Cancer Inst. 2004;96:1051-1053.

21. Paramsothy P, Jamieson DJ, Heilig CM, et al. The effect of highly active antiretroviral therapy on human papillomavirus clearance and cervical cytology. Obstet Gynecol. 2009;113:26-31.

22. Saslow D, Solomon D, Lawson HW, et al. American Cancer Society, American Society for Colposcopy and Cervical Pathology, and American Society for Clinical Pathology screening guidelines for the prevention and early detection of cervical cancer. J Low Genit Tract Dis. 2012;16(3):175-204.

23. Moyer VA; Preventive Services Task Force. Screening for cervical cancer: US Preventive Services Task Force recommendation statement. Ann Intern Med. 2012;156(12):880-891.

24. Denny L, Kuhn L, De Souza M, Pollack AE, Dupree W, Wright TC Jr. Screen-and-treat approaches for cervical cancer prevention in lowresource settings: a randomized controlled trial. JAMA. 2005;294: 2173-2181.

25. Sankaranarayanan R, Esmy PO, Rajkumar R, et al. Effect of visual screening on cervical cancer incidence and mortality in Tamil Nadu, India: a cluster-randomised trial. Lancet. 2007;370:398-406. 
26. Mwanahamuntu MH, Sahasrabuddhe VV, Pfaendler KS, et al. Implementation of 'see-and-treat' cervical cancer prevention services linked to HIV care in Zambia. AIDS. 2009;23(6):N1-N5.

27. Pfaendler KS, Mwanahamuntu MH, Sahasrabuddhe VV, Mudenda V, Stringer JS, Parham GP. Management of cryotherapy-ineligible women in a "screen-and-treat" cervical cancer prevention program targeting HIV-infected women in Zambia: lessons from the field. Gynecol Oncol. 2008;110(3):402-407.
28. Sankaranarayanan R, Nene BM, Shastri SS, et al. HPV screening for cervical cancer in rural India. $N$ Engl J Med. 2009;360(14): 1385-1394.

29. Keller MJ, Burk RD, Xie X, et al. Risk of cervical precancer and cancer among HIV-infected women with normal cervical cytology and no evidence of oncogenic HPV infection. JAMA. 2012;308(4): $362-369$.

\section{Publish your work in this journal}

The International Journal of Women's Health is an international, peerreviewed open-access journal publishing original research, reports, editorials, reviews and commentaries on all aspects of women's healthcare including gynecology, obstetrics, and breast cancer. The manuscript management system is completely online and includes a very quick and fair peer-review system, which is all easy to use. Visit http://www.dovepress.com/testimonials.php to read real quotes from published authors.

Submit your manuscript here: http://www.dovepress.com/international-journal-of-womens-health-journal 